\title{
Numerical Simulation of Flow Field in the Plate-Fin Reactor
}

\author{
Xian Zhang \\ School of Mechanical Engineering, Huaihai Institute of Technology, Lianyungang 222005, China. \\ zhaomg@hhit.edu.cn
}

Keywords: Simulation, two-phase fluid, reactor.

\begin{abstract}
On the basic of the PIV flows field measurement, mature commercial software to fit and test heat plate reactor momentum transfer mathematical model are used, and the flow field in various operation conditions of heat plate bioreactor is simulated. The transfer process of two-phase flow is complicated, we use the ideal even bubbles model to simulate, analyze and calculate, the deviation values of flow profile of two-phases are minor, but they are high than that of homogeneous phase flow. The more complicated the reactor configuration is, the more great the difference between mathematical model of reactor and actual process is, because we have to make much hypothesis on the model, so the behavior to confirm intricate boundary condition and partition it with meshes is more difficult, the deviation value of flow profile is to increases.
\end{abstract}

\section{Introduction}

The reactor can be divided into mechanical stirring reactor, a mechanical agitated reactor according to the types of energy inputting [1]. Non mechanical agitated reactor has more rapid development in recent years, mainly including the bubbling tower reactor, jet loop reactor, gas lift loop reactor and so on, but the technology is not yet mature, application is not wide. Mechanical agitating reactor has a long industrial application experience and a series of modular design, so it is used most widely [2].

Heat transfer modes such as traditional jacket, snake pipe, tube, and the tank outer wall spraying are generally used in the heat transfer devices of mechanical stirring type bioreactor. All these ways exist common faults :(1) the reactor requires a large number of heat exchange area;(2) the reactor temperature distribution is not uniform;(3) amplifier of the reactor limited by heat transfer; (4) the large amount of cooling water.

Heat plate reactor, as a new type of an agitated tank bioreactor, based on the working principle of a heat pipe, have the advantage of uniform temperature, high-heat transfer efficiency, temperature sensitivity, cooling water consumption, etc., and it solves many heat transfer problems of the traditional stirring type bioreactor [3].

Heat plate is a kind of high efficient heat transfer unit which uses both boiling and condensation phase change heat transfer. In the heat transfer process, due to the bubble turbulent, condensed droplets flow and high latent heat of phase change, so the thermal resistance is very low, thermal current intensity is very high, the thermal conductivity of heat pipe can reach hundreds of times or even thousands of times of that of copper.

In this paper, simulations of fluid velocity distribution of impeller area cross-section $\left(\mathrm{S}_{1}\right)$, discharge flow area cross-section $\left(\mathrm{S}_{2}\right)$ and main circulation area cross sectional $\left(\mathrm{S}_{3}\right)$ are done by using CFD software. Based on the two-phase fluid as the research system, the practice of using the mature CFD commercial software to solve and analyze the transfer process in the heat plate reactor, and of using the measured field data to fit the calculation results of momentum transfer process will provide the reliable momentum balance equation for the establishment and amplification of reactor mathematical model.

\section{Description of the Installation}


In this paper, the system testing flow profile of fluid in new reactor and heat plate wall is set up (see Fig 1and Fig 2); we measure and study effectively the whole new reactor's flow profile.

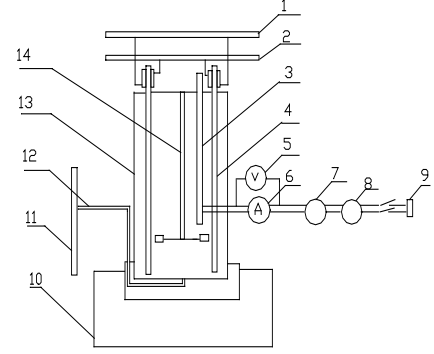

Fig.1 The research on heat transfer in heat plate reactor

1. Condensate water inlet pipe; 2 .Condensate water outlet pipe; 3 . Electric heating rod; 4 . Heat plate (three); 5. Voltage meter; 6. Current meter; 7. Regulator; 8. Voltage regulator; 9. Power supply; 10. Kettle body supporting; 11 . Compressed air plate; 12 . Inlet vent plate ;13.Reactor body;14 .Agitator kettle

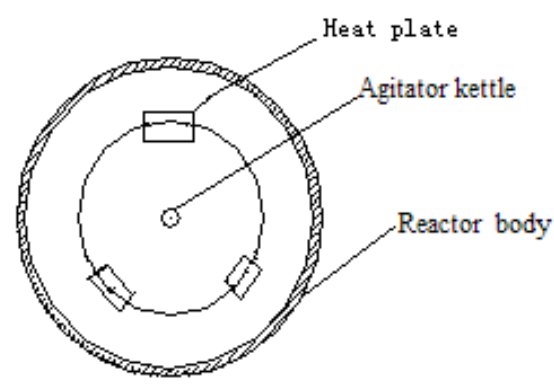

Fig.2 Distribution of three heat plates

\section{Heat Plate Reactor Model}

Momentum, mass balance equations. In general the stirred-tank reactor fluid flow can be assumed: (1) the time-averaged movement of fluid in the reactor is the steady state without change with the time (ignoring the efforts of periodic motion to fluid macroscopic motion in the stirred tanks [4]; (2) the fluid is continuous and incompressible. The flow characteristic is isotropy in the turbulent flow.

According to the velocity, the fluid flow is divided into laminar flow state and turbulent flow state. Even though in the same reactor, due to the fluid velocity distribution, it can cause certain region to be laminar flow, some to be turbulent flow. In these two kinds of flow conditions, we can derive the different quality of the reactor, and the momentum balance equation as follows [5-9]. The balance equation is used when it is the turbulent flow.

Turbulent flow is very complex; its flow pattern varies with time. Generally the instantaneous value of each parameter in the turbulent flow (such as the instantaneous value of velocities at various moments) varies. In order to facilitate the analysis and processing, we often use the average-time value (such as mean average velocity) of the parameters in a certain period of time. Apply the average-time value of "Reynolds stress" in the turbulent flow to the laminar quality, momentum balance equation, we can obtain the mathematical model in the turbulent flow:

(1) The basic balance equation

Mass balance equations of turbulent flow are same with turbulent flow of mass balance equations.

$$
\frac{\partial \boldsymbol{\rho}}{\partial \boldsymbol{t}}+\nabla \bullet(\boldsymbol{U})=\mathbf{O}
$$

Consider Reynolds stress mean item in the N-S formula, then we can get the momentum balance equation:

$$
\frac{\partial}{\partial t} \rho \mathbf{U}+\nabla \bullet(\rho \mathbf{U} \otimes \mathbf{U})=\mathbf{B}+\nabla \bullet(\sigma-\rho \overline{\mathbf{u} \otimes \mathbf{u}})
$$

Reynolds stress mean: 


$$
\frac{1}{\sqrt{\boldsymbol{g}}} \frac{\partial}{\partial t} \sqrt{\boldsymbol{g}} \rho+\nabla \cdot\left(\rho\left(\mathbf{U}-\frac{\partial z}{\partial t}\right)\right)=0
$$

Because we use the sliding grid to calculate the stirring paddle area, software CFX4.3 introduces the grid sliding velocity (prescribed stirring shaft is the $\mathrm{Z}$ ) to modify and transfer the transfer equation into:

$$
\begin{aligned}
& \frac{\mathbf{1}}{\sqrt{\boldsymbol{g}}} \frac{\partial}{\partial \boldsymbol{t}} \sqrt{\boldsymbol{g}} \boldsymbol{\rho}+\boldsymbol{-}-\left(\rho\left(\mathbf{U}-\frac{\partial \mathbf{z}}{\partial \boldsymbol{t}}\right)\right)=\mathbf{O} \\
& \frac{1}{\sqrt{g}} \frac{\partial}{\partial t} \sqrt{g} \rho \mathbf{U}+\nabla \cdot\left(\rho\left(\mathbf{U}-\frac{\partial z}{\partial t}\right) \otimes \mathbf{U}\right)=\mathbf{B}+\nabla \bullet \sigma \\
& \text { The fluid rotation around the Z-axis's: } \\
& \mathbf{B}=-2 \rho \omega \times \mathbf{U}-\rho \omega \times \omega \times \mathbf{z} \\
& \text { (2) The standard model }
\end{aligned}
$$

The standard model is a general model of solving turbulent viscosity in turbulent flow.

Turbulent viscosity model in turbulent:

$\mu_{T}=\mathrm{C}_{\mu} \rho \frac{k^{2}}{\varepsilon}$

Parameters $\mathrm{k}$ and $\varepsilon$ are respectively functions of the reactor space time coordinates and corresponding partial differential equations:

$$
\begin{aligned}
& \frac{\partial}{\partial t} \rho k+\nabla \cdot(\rho \mathbf{U} k)-\nabla \cdot\left(\left(\mu+\frac{\mu_{T}}{\sigma_{k}}\right) \nabla k\right)=P+G-\rho \varepsilon \\
& \frac{\partial}{\partial t} \rho \varepsilon+\nabla \cdot(\rho \mathbf{U} \varepsilon)-\nabla \cdot\left(\left(\mu+\frac{\mu_{T}}{\sigma_{\varepsilon}}\right) \nabla \varepsilon\right)=\mathrm{C}_{1} \frac{\varepsilon}{k}\left(P+\mathrm{C}_{3} \max (G, 0)\right)-\mathrm{C}_{2} \rho \frac{\varepsilon^{2}}{k}
\end{aligned}
$$

Make

$\mu_{\text {eff }}=\mu+\mu_{T}$

The role of the fluid pressure $\mathrm{P}$ is defined as:

$$
P=\mu_{\text {eff }} \nabla \mathbf{U} \cdot\left(\nabla \mathbf{U}+(\nabla \mathbf{U})^{\mathrm{T}}\right)-\frac{2}{3} \nabla \cdot \mathbf{U}\left(\mu_{\text {eff }} \nabla \cdot \mathbf{U}+\rho k\right)
$$

And

$G=G_{\text {buoy }}+G_{\text {rot }}+G_{\text {res }}$

Because $G_{\text {rot }}$ and $G_{\text {res }}$ in type (12) tend to 0, so the:

$\mathrm{G}=\mathrm{G}_{\text {buoy }}=-\frac{\mu_{T}}{\rho \sigma_{\rho}} g \cdot \Delta p$

The standard model can make the type (2) momentum equation transform into:

$\frac{\partial}{\partial t} \rho \mathbf{U}+\nabla \cdot(\rho \mathbf{U} \otimes \mathbf{U})-\nabla \cdot\left(\mu_{\text {eff }} \nabla \mathbf{U}\right)=-\nabla p+\nabla \cdot\left(\mu_{\text {eff }}(\nabla \mathbf{U})^{T}\right)+\mathbf{B}$

In a wide range of Reynolds number, with the standard model, the solved turbulent flow error is slightly high, so we have carried out the models of a higher accuracy in the low turbulence and turbulent zone.

(3) The model at low Reynolds number

When $5000 \leqslant \operatorname{Re} \leqslant 30000$, the low Reynolds number model:

$\mu_{T}=\mathrm{C}_{\mu} f_{\mu} \rho \frac{k^{2}}{\varepsilon}$

The partial differential equation:

$\frac{\partial}{\partial t} \rho k+\nabla \bullet(\rho \mathbf{U} k)-\nabla \bullet\left(\left(\mu+\frac{\mu_{\mathrm{T}}}{\sigma_{k}}\right) \nabla k\right)=P+G-\rho \varepsilon-D$

$\frac{\partial}{\partial t} \rho \varepsilon+\nabla \cdot(\rho \mathbf{U} \varepsilon)-\nabla \cdot\left(\left(\mu+\frac{\mu_{\mathrm{T}}}{\sigma_{\varepsilon}}\right) \nabla \varepsilon\right)=C_{1} \frac{\varepsilon}{k}\left(P+C_{3} \max (G, 0)\right)-C_{2} \rho \frac{\varepsilon^{2}}{k}+E$

And 


$$
\begin{aligned}
& f_{\mu}=\exp \left(\frac{-3.4}{\left(1+\frac{R_{T}}{50}\right)^{2}}\right), \quad R_{T}=\frac{\rho k^{2}}{\mu \varepsilon} \\
& D=2 \mu\left(\Delta k^{\frac{1}{2}}\right)^{2} \\
& \sigma_{\varepsilon}=\frac{k^{2}}{\left(C_{1}-C_{2}\right) \sqrt{C_{\mu}}} \\
& E=2 \frac{\mu \mu_{T}}{\rho}(\Delta \mathbf{U})^{2}
\end{aligned}
$$

(4) The RNG model of the high Reynolds number

When $\mathrm{Re}>$ 30000, the high Reynolds number model (RNG model) is as follows:

$$
\mu_{T}=\mathrm{C}_{\mu} \rho \frac{k^{2}}{\varepsilon}
$$

The partial differential equation:

$$
\begin{aligned}
& \frac{\partial}{\partial t} \rho k+\nabla \cdot(\rho \mathbf{U} k)-\nabla \bullet\left(\left(\mu+\frac{\mu_{T}}{\sigma_{k}}\right) \nabla k\right)=P+G-\rho \varepsilon \\
& \frac{\partial}{\partial t} \rho \varepsilon+\nabla \cdot(\rho \mathbf{U} \varepsilon)-\nabla \cdot\left(\left(\mu+\frac{\mu_{\mathrm{T}}}{\sigma_{\varepsilon}}\right) \nabla \varepsilon\right)=\left(\mathrm{C}_{1}-\mathrm{C}_{\mathrm{RRNG}}\right) \frac{\varepsilon}{k}\left(P+\mathrm{C}_{3} \max (G, 0)\right)-\mathrm{C}_{2} \rho \frac{\varepsilon^{2}}{k}
\end{aligned}
$$

But for

$$
\begin{aligned}
& \mathrm{C}_{1 R N G}=\frac{\eta\left(1-\frac{\eta}{\eta_{0}}\right)}{1+\beta \eta^{3}}, \\
& \eta=\left(\frac{p_{s}}{\mu_{r}}\right)^{0.5} \frac{k}{\varepsilon}
\end{aligned}
$$

The geometry model. The geometric model is geometric figure of heat plate bioreactor for numerical calculation meshes division in the CFX software. CFX software produces line from the point, then produce area and body. Therefore it has drawn heat plate bioreactor geometry model, and according to this geometric model with mesh generation, it has introduced geometric files in the computing program: “*.geo”.

Calculation method. Fluid mechanic research methods, according to different riddling partition technology, can be divided into: Euler method, Lagrange method and the ALE method. The Euler method investigates the change of the fluid under the fixed reference coordinate system. It can calculate fluid flow problems with large distortions in a multidimensional space, but the fluctuating interface cannot be clearly identified; Lagrange methods block the grid in the fluid, and make the grid move with the fluid. It can evidently determine fluid interface and the reaction fluid internal details. Nevertheless, it cannot deal with the shipping and large distortion. It is easy to cause the grid intersection leading the calculation cannot be normal; ALE method combines Euler method and Lagrange method together, which can mix mesh as the Euler method, and make the grid change in any way, so it cannot be only copious flow distortion problems but also provide a fluid movement detail. This mesh has excellent technology adaptability [10-12]. This paper basically uses the method of ALE. The exact grid division method uses the staggered grid system. For the complex geometry of the mesh division, Peric M. [11, 12] had put forward the collocated grid method.

In this paper, we use computational fluid dynamics software CFX4.3. For the particularly complex rotary impeller region in the reactor, we also apply the moving grid method ${ }^{[11,13]}$. This method will divide the computational domain into two parts (see Fig.3). One part is "inside mobile area (a) " containing motor blades; the other is "external static area (b) " containing static baffle plate and the 
groove. The two parts between the grids are required independent of each other. This is realized through the establishment of mismatched boundary conditions in the CFX4.3 software.

Command File in the establishment of a mismatched boundary condition to realize. The External (b) grid is a static, internal (a) grid rotates together with the stirring paddle, and the center rotating grid is the impeller shaft (Fig.4). Two parts through the sliding interface between grids conduct interpolation processing. Because the computing amount of sliding grid is very large, in order to save time, at the beginning of computing, we select a larger time step to eliminate the initial effect, and then select a smaller time step to obtain the required precision.

Make the appropriate time averaged process for the instantaneous value of the flow field calculated by the moving mesh method to obtain the theoretical calculation value which can compare with the experiment values in the steady-state flow experiment.

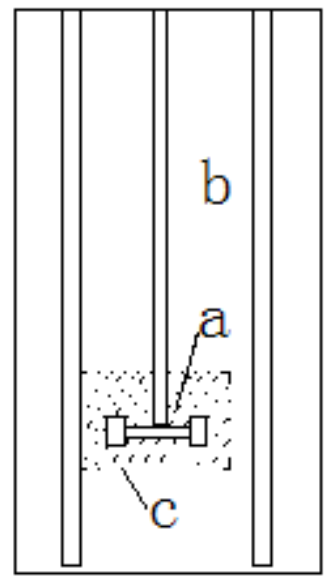

Fig.3.Sketch map of sliding mesh

a. Internal moving zone, b. external static area, c. moving boundary.

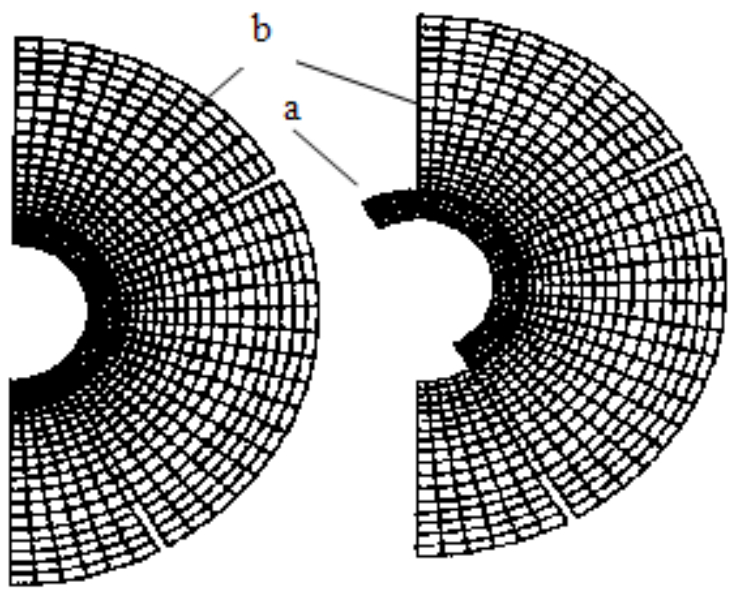

Fig.4 Sketch map of mesh's sliding

A .the grid of initial positions, B. moving grid changed with the time.

\section{Result and Discussion}

When the mixing speed is $\mathrm{n}=220 \mathrm{R} / \mathrm{min}$, the flow in heat plate bioreactor is in the low turbulent state. Using CFX commercial software to calculate the velocity distribution in $\mathrm{X}$ and $\mathrm{Y}$ directions and axial velocity distribution in the $\mathrm{Z}$ direction in the reactor. Use CFX commercial software to calculate the velocity $\left(\mathrm{U}_{\mathrm{X}}, \mathrm{U}_{\mathrm{Y}}\right.$, and $\mathrm{U}_{\mathrm{Z}}$ ) distribution of $\mathrm{X}$ direction, $\mathrm{Y}$ direction and reactor axial (Z) cross section in the reactor. The calculated values of $U_{X}, U_{Y}$ corresponded to $U_{X}$ and $U_{Y}$ of measured cross section . Resultant velocity is radial velocity of $U_{X}$ and $U_{Y}$ of the measured cross section flow field in the PIV system. The simulation calculation Uz corresponds to three representative cross 
section flow field in the reactor to simulate calculation and discuss: the first cross-sectional area $\mathrm{S}_{1}$ located in the fluid impeller area $(\mathrm{H}=53.2 \mathrm{~mm})$, second cross-sectional $\mathrm{S}_{2}$ located in the fluid discharge area $(\mathrm{H}=42.4 \mathrm{~mm})$, the third cross section $\mathrm{S}_{3}$ locates in fluid main circulation area $(\mathrm{H}=$ $63.9 \mathrm{~mm}$ ).

The velocity distribution simulation at cross sectional of the impeller region $\left(S_{1}\right)$. On the intersection line of $S_{1}$ cross-sectional and longitudinal $V_{1}$ in the reactor, the velocity simulation value of $\mathrm{Ux}$, Uy and Uz of every particle is shown in Fig.5 Fig.7.

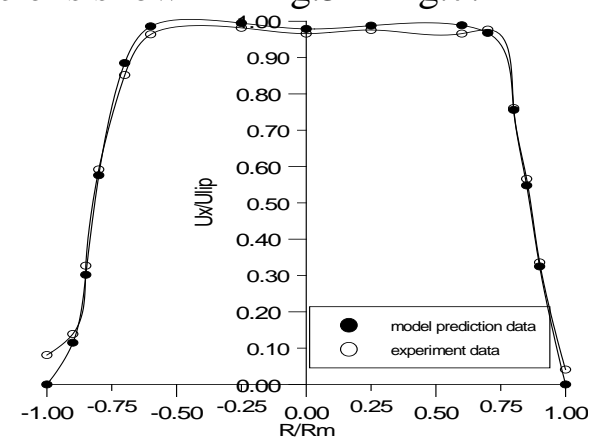

Fig.5 Profile of $\mathrm{X}$ velocity for $\mathrm{S}_{1}$ cross section

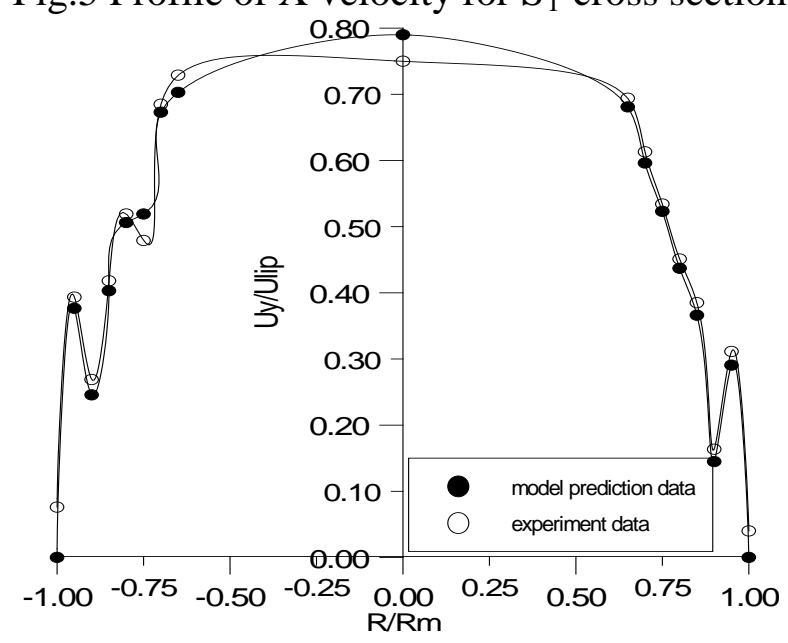

Fig.6 Profile of $\mathrm{Y}$ velocity for $\mathrm{S}_{1}$ cross section

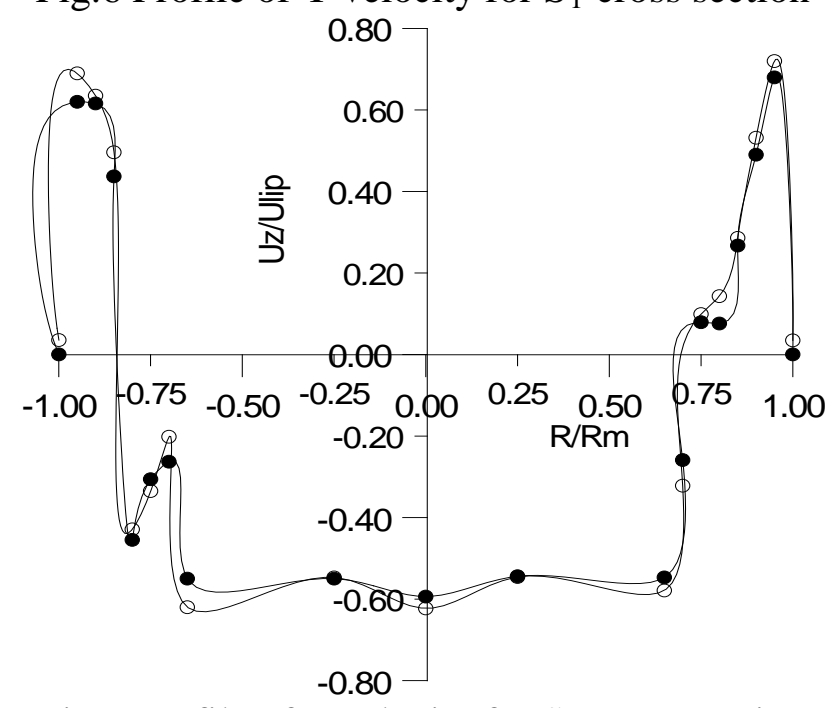

Fig.7 Profile of $\mathrm{Z}$ velocity for $\mathrm{S}_{1}$ cross section

Under this conduction, the flow velocity simulation calculation value of impeller area is identical to the experimental value. The average error between calculated values and experimental values of $\mathrm{X}$ position velocity component is 5.3\%, the average error of $\mathrm{Y}$ and $\mathrm{Z}$ position are only $9.1 \%$ and $10.5 \%$ respectively. The error of calculated value and the measured value of Y position velocity component at the area $\mathrm{R} / \mathrm{Rm}=0.59$ is largest (15.5\%). Compared with the homogeneous fluid, its error of radial 
velocity calculation value is higher than experimental value of error by $10 \%$, and the simulation error of axial velocity is higher by $100 \%$.

Cross sectional velocity distribution simulation in discharge flow area $\left(S_{2}\right)$. On the intersection line of S cross-sectional and longitudinal V1 in the reactor the velocity simulation value of Ux, Uy and Uz of every particle is shown in Fig.8 Fig. 10 .

Under this conduction, the flow velocity simulation calculation value of drainage area is identical to the experimental value. The average error between simulations calculated values and experimental values of $\mathrm{X}$ position velocity component is $9.2 \%$, the average error of $\mathrm{Y}$ and $\mathrm{Z}$ position are only $8.5 \%$ and $7.3 \%$ respectively. The error of calculated value and the measured value of Y position velocity component at the area $\mathrm{R} / \mathrm{Rm}=0.88$ is largest $(15.5 \%)$.

The fluid at turbulent flow state is similar with homogeneous fluid at this cross section, but the flow field is more complex.

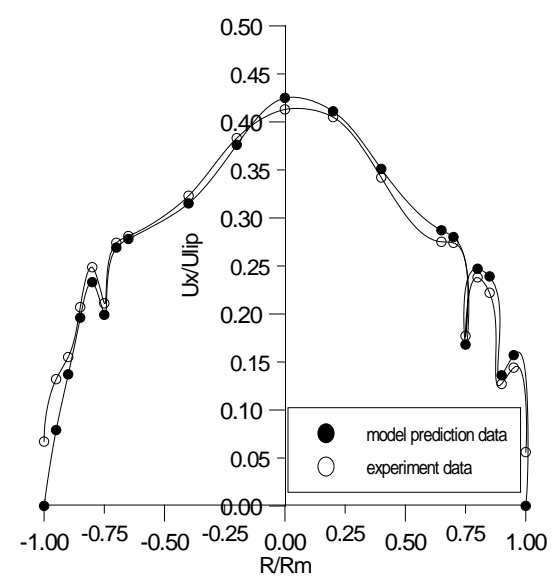

Fig.8 Profile of $\mathrm{X}$ velocity for $\mathrm{S}_{2}$ cross section

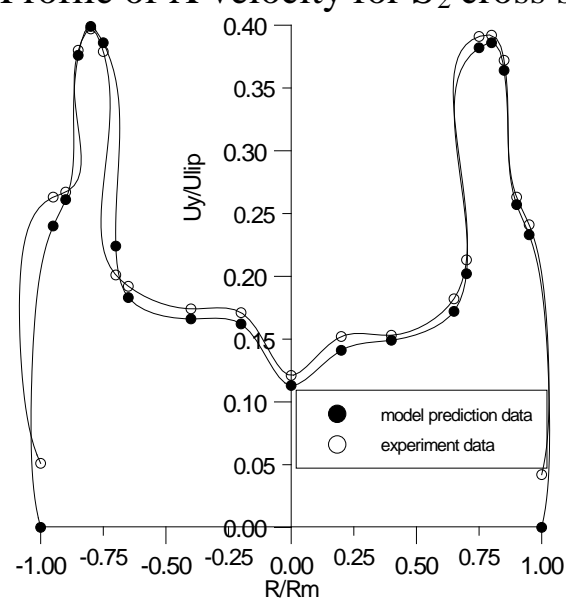

Fig.9 Profile of $\mathrm{Y}$ velocity for $\mathrm{S}_{2}$ cross section

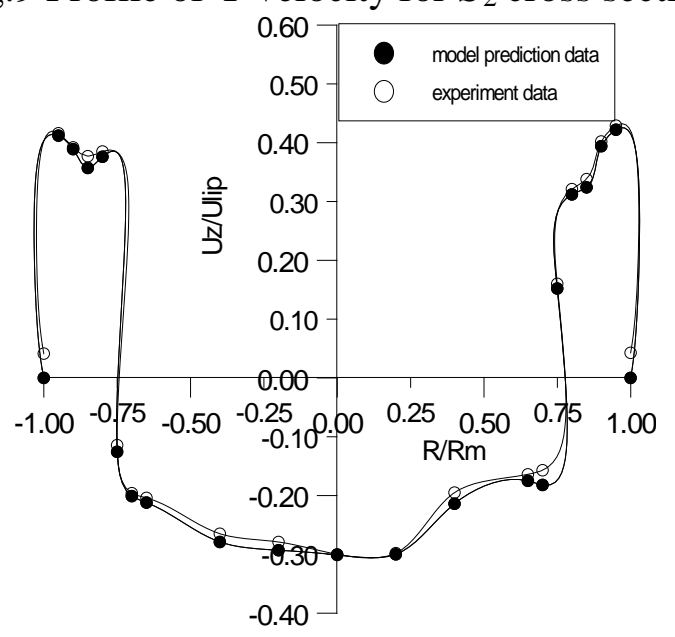

Fig.10 Profile of $Z$ velocity for $S_{2}$ cross section 
Cross sectional velocity distribution simulation at main circulation area $\left(\mathbf{S}_{3}\right)$. On the intersection line of $S_{3}$ cross-sectional and longitudinal $V_{1}$ in the reactor the velocity simulation value of $\mathrm{Ux}$, Uy and $\mathrm{Uz}$ of every particle is shown in Fig.11 -13.

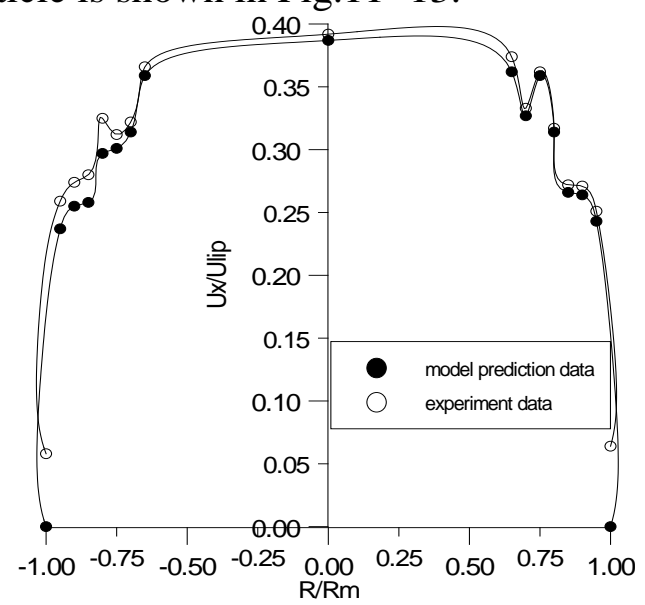

Fig.11 Profile of $\mathrm{X}$ velocity for $\mathrm{S}_{3}$ cross section

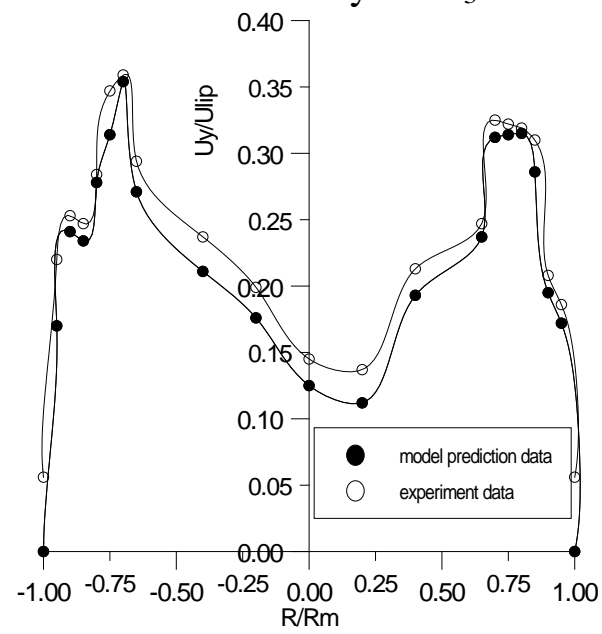

Fig.12 Profile of $\mathrm{Y}$ velocity for $\mathrm{S}_{3}$ cross section

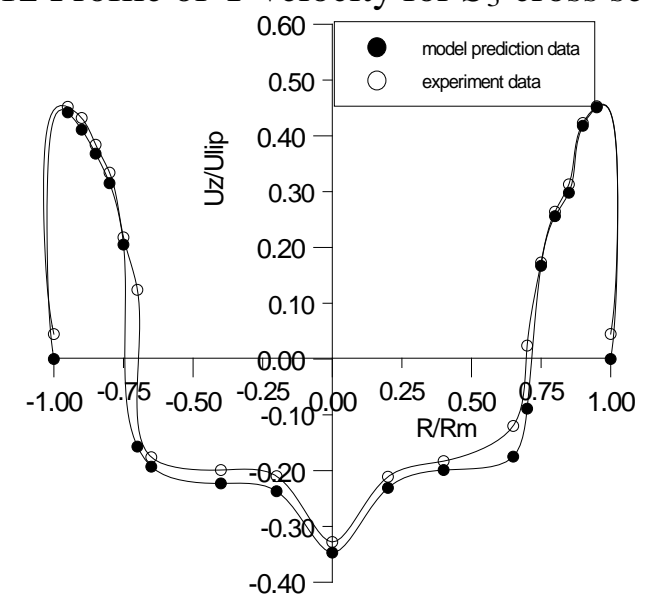

Fig.13 Profile of $\mathrm{Z}$ velocity for $\mathrm{S}_{3}$ cross section

Under this conduction, the flow velocity simulation calculation value of main circulation area is identical to the experimental value. The average error between calculated values and experimental values of $\mathrm{X}$ position velocity component is $8.4 \%$, the average error of $\mathrm{Y}$ and $\mathrm{Z}$ position are only $10.3 \%$ and $7.8 \%$ respectively. The error of calculated value and the measured value of $\mathrm{Y}$ position velocity component at the area $\mathrm{R} / \mathrm{Rm} \approx 0.65$ is largest(15.4\% ). The fluid at turbulent flow state is similar with homogeneous fluid at this cross section.

\section{Conclusion}


The fluid in heat pipe bioreactor with mixing speed $n=220 \mathrm{r} / \mathrm{min}, \quad \mathrm{Ug}=1 \mathrm{~L} / \mathrm{min}$ of two phase fluid (H2O-Air) is a kind of very complex turbulent fluid. Compared with laminar flow, the velocity gradient and the flow state are similar with each other. Reactor fluid radial, axial velocity component simulation calculation was in agreement with experimental data, the average error is $8.5 \%, 10.6 \%$, which explores the maximum error is $15.5 \%$. Two phase fluid transfer process is very complex, and the bubble random behavior is difficult to determine and describe, therefore, we use ideal uniform bubble model to simulate, analysis and calculate the result. Because of the effect of the bubbles, the value of the flow field is not stable and accurate; the average error between the calculation value and experimental value is higher than the homogeneous fluid.

\section{References}

[1] K. Babita, S. Sridhar, K.V. Raghavan, Membrane reactors for fuel cell quality hydrogen through WGSR - Review of their status, challenges and opportunities, International Journal of Hydrogen Energy. (2011) 6671-6688.

[2] Ranade V V, Dommeti S M S, Computational snapshot of flow generated by Z impellers in baffled stirred vessels, Trans I ChemE. 74 (2009) 476-478.

[3] Harvey P S, Greaves M, Turbulent flow in an agitated vessel, Trans I ChemE. 60 (2010) 202-208.

[4] Gaskell P H, Lou AKC, Simulation of flow in stirred vessels with Z flow impel, Int J Num Meth Fluids. 8 (2009) 617-625.

[5] Wu H, Patterson G K, Laser-Doppler measurement of turbulent flow parameters in a stirred mixer, Chem Eng Sci. 44 (2009) 2210-2215.

[6] Brucato A, Ciofalo M,Grisafi F,etal, Numerical prediction of flow fields in baffled stirred vessels: a comparison of alterative modeling approaches, Chem Eng Sci. 53 (1998) 3680-3684.

[7] Schafer M,Yu J, Genenger B, et al, Turbulence generation by different types of impellers, Proceeding of 10th European Conference on Mixing. Delft:Delft University of Technology, (2000) 9-15.

[8] Yianneskis $\mathrm{M}$, Whitelaw $\mathrm{J} \mathrm{H}$, On the structure of the trailing votices around Rushton turbine blades, Trans I ChemE. 71A (2009) 346-348.

[9] Revstedt J, Fuchs L, Tragardh C, Large eddy simulation of the turbulent flow in a stirred reactor, Chem Eng Sci. 53 (1998) 4045-4050.

[10] Rhie C M, Chow W L, A numerical study of the turbulent flow past an aerofoil with trailing edge separation, AIAA J. 21 (1983) 1526-1530.

[11] Ng K, Fentiman N J, Lee K C, et al, Assessment of sliding mesh CFD predictions and LDA measur-ement of the flow in a tank stirred by a Rushton impeller, Trans I ChemE. 76A (1998) 738-742.

[12] Lee K C, Ng K, Yanneskis M, Sliding mesh predictions of the flow around Rushton impellers, I ChemE Symp Ser. 140 (2009) 48-56.

[13] Suzanne M K, Philip E W, The mean flow field produced by a 45 degree pitched balde turbine, Canad J ChemE. 71 (1993) 42-46.

[14] Y. Chang, An intelligent analysis and verification model for consistent SMIL presentations, JCIT: Journal of Convergence Information Technology. 7 (2012) 332-341.

[15] Zhang Qing-hui, Cheng Heng-feng, Xu Hong, Research of frame size and group division in anti-collision algorithms of RFID, IJACT. 3 (2011) 9-14. 